\title{
Reflexiones en torno al sentido de la vida en los espacios áulicos
}

\author{
Insights on the Meaning of Life in the Classroom
}

\author{
Giselle Miranda-Cervantes ${ }^{1}$ \\ Universidad Nacional \\ División de Educación Básica Centro de Investigación y Docencia en Educación \\ Heredia, Costa Rica \\ giselle.miranda.cervantes@una.cr
}

Recibido 7 de noviembre de 2013 • Corregido 28 de mayo de 2014 • Aceptado 22 de agosto de 2014

Resumen. Este artículo parte de la premisa de que la convivencia en los espacios áulicos debe ser una dinámica emocional compartida dentro de un espacio de respeto mutuo del sí mismo, del otro, de la otra y de cada uno de sus quehaceres. Propone que en estos espacios saludables se debe gestar la transformación de todas las personas participantes, desde una perspectiva compleja en la que los procesos vitales y los procesos de aprendizaje sean anidados en un mismo proceso. Pretende alertar acerca de la urgente necesidad de devolverle el verdadero encanto a la educación, replanteando el concepto y el sentido de la vida, para poder saborear la existencia desde un nuevo paradigma en el que se trabaje con el intelecto, con el corazón y con la vida misma, en un estado superior de armonía.

Palabras claves. Aprendizaje, aula, biopedagogía, complejidad, convivencia, incertidumbre, lenguajear, sentido, transformación, vida.

Abstract. This article is based on the premise that coexistence in classrooms must be an emotional dynamic shared within a space of mutual respect for oneself, one another, and each other's activities. The paper proposes that the transformation of the participants must be developed in these healthy spaces, based on a complex perspective, in which the vital and learning processes are nestled in one single process. It also strives to alert on the urgent need to recover education's true charm, by reconsidering the concept and meaning of life, in order to savor existence from a new paradigm where people work with their intellect, their heart, and life itself, in a higher state of harmony.

Keywords. Learning, classroom, biopedagogy, complexity, coexistence, uncertainty, language creation, purpose, transformation, life.

${ }^{1}$ Doctora en Educación con especialidad en Mediación Pedagógica. Bachiller, licenciada y máster en Psicología de la Universidad de Costa Rica. Labora como académica e investigadora desde 1990 en la División de Educación Básica del Centro de Investigación y Docencia en Educación (CIDE) de la Universidad Nacional de Costa Rica, unidad académica en la que ocupó los cargos de directora, miembro del Consejo Académico y coordinadora de las carreras de Educación Especial y de Enseñanza del Inglés para I y II ciclos. Su interés en el campo de la investigación destaca las áreas de diversidad, seguimiento a egresadas de la carrera de Educación Especial, relaciones interpersonales, clima social de aula y pedagogía saludable. 
doi: http://dx.doi.org/10.15359/ree.18-3.16

URL: http://www.una.ac.cr/educare

CORREO: educare@una.cr

El sentido no se traspasa, ni se enseña; el sentido se vive, se construye, se hace y se rehace viviendo a plenitud y conscientemente el proceso...

Gutiérrez (2006)

\section{Transformación en la convivencia}

Durante más de veinte años, he tenido la oportunidad de compartir "espacios de aula" con muchachas y muchachos que inician su proceso de formación profesional universitaria. Lo que, en sí mismo, ha sido para mí una excelente oportunidad para revisar mis anteojos para "mirar la vida" y para relacionarme con las demás personas. No obstante, lo más interesante de este proceso ha sido la transformación que, casi sin darme cuenta, se ha gestado en mí como persona y en diferentes áreas de mi vida. Retomando las palabras de Maturana (2002), este proceso ha sido para mí, efectivamente, una "transformación en la convivencia".

Inicié mi experiencia docente hace más de dos décadas en la Universidad Nacional de Costa Rica (UNA), institución que me contrató por mi formación en el área de la psicología para impartir lecciones en las carreras dirigidas a futuros y futuras profesionales en educación. Mis planeamientos y la preparación de cada una de mis lecciones fueron rigurosos y me sentía satisfecha cuando al terminar la lección, la pizarra semejaba una gran colcha de quilting repleta de pedacitos de tela representados por fórmulas, conceptos, gráficos y procedimientos, los cuales esperaba que hubiesen sido fielmente copiados en cada uno de los cuadernos de mis estudiantes. Observar que el polvo de la tiza se acumulaba hasta en mis pestañas, era la mejor forma de "sentir" que estaba "enseñando" lo que mis estudiantes "tenían que aprender". Por supuesto que no puedo quitarle protagonismo al paradigma tradicional dentro del cual me estaba moviendo, ni desacreditar el conocimiento científico que estaba "transmitiendo".

Todo este proceso se hizo acompañar por la inevitable evaluación escrita que me permitía comprobar que el "esfuerzo había valido la pena". Un promedio de 75 en adelante me hacía sentir bien, porque usualmente el resto de las calificaciones se distribuían de acuerdo con la famosa campana de Gauss, lo que significaba que mis estudiantes mostraban una distribución normal, es decir, algunos pocos muy altos, algunos pocos muy bajos, los intermedios altos, los intermedios bajos y un porcentaje importante, "coqueteando" con la conocida media estadística. Estos números me dieron, durante los primeros años como docente universitaria, un sentimiento de ser "buena profesora".

No obstante, conforme pasó el tiempo, la convivencia con mis estudiantes me permitió sentir que este espacio relacional es mucho más que una serie de contenidos para transmitir, que ni siquiera puedo pretender enseñar aquello que a mis estudiantes no les interesa aprender y que todo aquello que compartimos durante dos, tres o cuatro lecciones semanales, siempre va a estar permeado por vivencias mías, suyas y nuestras. Puedo compartir un conocimiento y mediar para que se genere un proceso individual y colectivo de aprendizaje; pero sin perder de vista que solo aquello que realmente tiene un significado para la persona será realmente aprendido. 
Maturana (2002) define claramente que"educar es crear, realizar, y validar en la convivencia, un modo particular de convivir. Esto siempre se realiza en una red de conversaciones que coordina el hacer y el emocionar de los participantes" (p. 147). En este mismo sentido, dicho autor define la educación como:

Proceso de transformación en la convivencia, [que] ocurre en todas las dimensiones relacionales del vivir, tanto de los espacios privados de la familia o del colegio, como en los espacios públicos de la calle, la televisión, el cine, el teatro o la radio, que, como redes [conversacionales] (el entrelazamiento del hacer y el emocionar), definen cotidianamente lo deseable y lo no deseable, lo legítimo y lo ilegítimo, lo hermoso y lo feo, lo honesto y lo deshonesto, lo aceptable y lo no aceptable ... en el convivir de la comunidad a [la que las personas] se incorporan. (Maturana, 2002, pp. 10-11)

Por tanto, la tarea de la educación, desde su punto de vista, “... es crear [espacios] de transformación en el convivir de las personas para el vivir" (p. 140).

Efectivamente, dichos conceptos solo pueden comprenderse, si esta forma de convivencia es vivida tanto en el aula como en los diferentes espacios relacionales que asumimos diariamente, ya sea como pareja, hijos, hijas, hermanos, hermanas, colegas, vecinos, vecinas, amigos y amigas. Esta debe ser una dinámica emocional compartida y circunscrita dentro de un espacio de respeto mutuo del sí mismo, del otro, de la otra y de cada uno de sus quehaceres. Es en estos espacios "saludables" donde se origina, efectivamente, la transformación de la persona, siempre y cuando visualicemos los procesos vitales y los procesos de aprendizaje como partes integrales dentro del propio proceso.

Según lo afirma Maturana (2002), "Ios niños aprendemos a generar el mundo que vivimos en la convivencia con los adultos con los cuales convivimos" (p. 141). El tejido de la vida propia, la del otro, la de la otra y las experiencias compartidas se transforma, así, en el fundamento esencial de la biopedagogía, por ser esta un nicho de vida, de aprendizaje y, por consiguiente, como lo señala Assmann (2002), los “... procesos vitales y [los] procesos cognitivos se han vuelto prácticamente sinónimos" (p. 26).

No obstante lo anterior, resulta interesante observar la transformación que en muchos casos muestran en sus aulas algunas de las personas que se dedican a la docencia, independientemente del nivel en el que se encuentren. El espacio áulico parece ser terreno particularmente fértil para "sacar" nuestras más ocultas necesidades de poder y dejarnos llevar por el Ego y por todo aquello que nuestra fragmentación "nos dice" que somos. Así, entramos al aula con este nombre, con esta apariencia, con estos títulos y con estos conocimientos. Es decir, nos presentamos ante el estudiantado con todos aquellos atributos y poses que nuestra fragmentación dice que somos; pero casi nunca mostramos lo que realmente somos.

Ante esta realidad, personalmente me resulta refrescante y significativo rescatar algunas de las ideas extraídas de los diálogos que Carlos Castaneda entabla con su célebre personaje 
doi: http://dx.doi.org/10.15359/ree.18-3.16

URL: http://www.una.ac.cr/educare

CORREO: educare@una.cr

de Don Juan, quien afirma que no existen ni maestros ni estudiantes cuando de conocimiento se habla, porque este no se puede transmitir y solo se llega a alcanzar mediante la experiencia individual y personal (Castaneda, 2001).

Dentro de esta misma perspectiva Maturana y Varela (1998) afirman que los procesos de comunicación van a depender más de lo que sucede con lo que se recibe que de lo que se entrega. En otras palabras, como docentes mediamos; pero no enseñamos y, usualmente, confundimos la transmisión de información, datos y contenidos, con los procesos de aprendizaje.

Nadie enseña y puede ser que ni siquiera los libros nos den el conocimiento que andamos buscando porque, como el mismo Castaneda (2001) lo ha expresado, en los libros no está el conocimiento y, por el contrario, se debe andar el camino para corroborar, en el vivir, ese conocimiento al que hacen referencia.

No escapa, entonces, de esta reflexión, la necesidad de destacar la importancia de las vivencias personales y colectivas que se generan en los procesos de aprendizaje y en todos los procesos que vivimos diariamente en el aula y en cualquiera de nuestros contextos. Según lo que plantea Maturana (2002), "... otra cosa fundamental y universal... es que ningún [individuo] está donde está por accidente ... [somos] resultado de una cierta historia de interacciones" (p. 98), y es en la interacción con su nicho vital que se gestan los procesos de aprendizaje.

No obstante lo anterior, en muchas ocasiones exponemos a nuestros niños, niñas y jóvenes a procesos totalmente desarmonizados, en los cuales separamos la parte cognitiva y racional, de las emociones y de las vivencias $y$, en este proceso, sin darnos cuenta, los docentes y las docentes también nos estamos auto-mutilando como seres integrales, al creer que podemos separar, en partes, a la persona que somos para así desempeñar un papel específico.

Como muy bien lo señala Assmann (2002), se requiere devolver realmente el encanto a la educación porque en este acto se encuentra "...en juego la autovaloración personal del profesorado, la autoestima de cada persona implicada [y, sobre todo porque] podemos estar siendo cómplices con el delito de un apartheid neuronal que, al no propiciar ecologías cognitivas, está, de hecho, destruyendo vidas" (p. 24).

Nuestros ancestros conocían muy bien su relación con la Tierra, con el cosmos y estaban sintonizados con la vida. Hoy día, por el contrario, se ofrece una educación "masiva" en la que se plantea que si quieres ser exitoso, debes caber dentro de los estándares que la sociedad espera de cada persona; olvidando reconocer cuáles son tus dones, tus sueños y tus talentos. Las personas tienen que "trabajar" y para ello olvidarse de ser artistas, compositoras o pintoras para transformar el desarrollo de estas habilidades en el sueño lejano que abandonaron en su infancia o en su adolescencia.

El tiempo lo hemos desvirtuado totalmente, ahora es lineal; no cíclico como nos lo indican las enseñanzas mayas. No despertamos cuando amanece sino cuando lo indica la alarma del reloj y no dormimos cuando se oculta el sol sino cuando apagamos el televisor. Olvidamos que 
el tiempo es una espiral ascendente que se renueva, es decir, que los ciclos vitales se repiten y regresan para que podamos aprender y evolucionar. Con ello, también perdimos la noción de nuestro lugar en el cosmos como seres eternos; utilizamos la fecha de nacimiento para definir nuestra existencia, lo que estuvo antes no interesa y, por tanto, lo obviamos. Perdimos la sabiduría de la vida que se repite en ciclos que retornan para que podamos evolucionar como seres universales y encontrar el porqué de la vida, el porqué vinimos aquí, qué hay antes y quiénes realmente somos.

Dentro de esta perspectiva, el ser humano se encuentra totalmente desarmonizado, aunque es parte de un sistema planetario en el cual todos los elementos fundamentales interactúan y recrean nuevos niveles de complejidad. En este sentido, diferentes autores hablan de la emergencia del orden o de la simultaneidad de orden-caos, para justamente identificar la autoorganización como:

La dinámica de aparición espontánea de patrones de orden y de caos en un sistema debido a las relaciones recursivas internas del propio sistema o a las interacciones del mismo entorno. Con la aparición de esas cualidades emergentes, aumenta la complejidad". (Assmann, 2002, p. 129)

No obstante, lejos de llegar a encontrar armonía en la dinámica cotidiana, el ser humano cada día se encuentra más desarmonizado con el entornoy consigo mismo. Se hace indispensable vaciarnos del ruido que nos ofrece la cotidianeidad, porque estamos en un momento en el cual no cabe ni una gota más en nuestro vaso sin riesgo de que este llegue a desbordarse. Ese es nuestro día a día y esa es la forma de "vida" que están aprendiendo nuestros niños, niñas y jóvenes en sus hogares, en sus barrios y en sus aulas. Debemos, por tanto, deshacernos de toda la basura que hemos recolectado y disponer de nuestro propio espacio para llenar el vacío, escuchar el silencio, la canción del viento, la voz de los animales, de las plantas y de toda la creación. Esto es lo que algunos han llamado la comunicación de espíritu a espíritu; sin máscaras, sin posturas, sin nada de lo que nuestro ego y nuestra fragmentación nos determina como ropajes con los que nos vestimos cuando interrelacionamos con nuestros estudiantes y nuestras estudiantes. Lo que hace falta es, precisamente, una comunicación llana, abierta, horizontal y armoniosa.

\section{La incertidumbre en el aula}

Le tenemos mucho miedo a la incertidumbre manifiesta en el aula, por esa razón nos protegemos llevando todo planeado, cronometrado y organizado; como modo de garantizar que nada quede al azar. Sin embargo, afirma Maturana (2002), el poder desaparece cuando desaparece el miedo al otro y, por el contrario, el poder surge cuando los otros hacen lo que yo quiero, es decir, lo que yo traigo preestablecido para ese día y ese momento de interrelación áulica. Encubrimos, en muchas de nuestras estrategias didácticas, diferentes formas de control para que, lo que llama Maturana el acto de reflexión, no se exprese. 
doi: http://dx.doi.org/10.15359/ree.18-3.16

URL: http://www.una.ac.cr/educare

CORREO: educare@una.cr

La pregunta del discente es básica desde el punto de vista de dicho autor, ya que conlleva a ese acto de reflexión, el cual es visto como un acto muy poderoso porque permite soltar sentimientos y con ellos también la certidumbre. Es, entonces, cuando podemos aceptar y hasta deleitarnos con el "fin de las certezas" (Assmann, 2002, p. 77). Este acto de reflexión, por tanto, permite un modo diferente de estar inmerso en otra reconfiguración relacional y posibilitar nuevas reflexiones individuales y colectivas; en otras palabras, permite la construcción colectiva de un sistema relacional nuevo.

En el caso de los niños y las niñas, a criterio de Maturana (2002), son seres que están abiertos a nuevos espacios relacionales porque no tienen respuestas hechas de antemano, como sí las tenemos las personas adultas (padres, madres y docentes). Lo que niega el escuchar es el tener respuestas hechas a toda pregunta y, por eso, el niño y la niña que no son escuchados sufren la negación de su propio ser.

De la misma manera, apunta Maturana (2002) que, solo si yo acepto la legitimidad del estudiante $y$, por tanto, la legitimidad de su pregunta, lo voy a tomar en cuenta como persona. Cuando un estudiante pregunta desde el desconocimiento de lo que su docente comparte, desde sus propias vivencias, desde un distinto saber y lo que dice es diferente a lo que yo estoy diciendo, se abre ante mis ojos una oportunidad reflexiva, en la cual nos escuchamos mutuamente.

Dentro de esta reflexión, Maturana (2002) destaca el lenguajear como forma de conversar y dar vueltas juntos en el encuentro. Concordantemente, cada palabra (sonido o gesto) no indica nada externo a nosotros, sino que es un elemento en el flujo de coordinaciones de coordinaciones de haceres y emociones que toman lugar en el vivir juntos en el lenguaje (Ruiz, 1997). El verbo lenguajear desde la perspectiva de Maturana (2002) enfatiza el carácter dinámico relacional del lenguaje; mientras que conversación se refiere "... al entrelazamiento de las coordinaciones de coordinaciones conductuales consensuales y las emociones que [sic] ocurre al vivir juntos en el lenguaje (Ruiz, 1997, La noción del lenguaje, párr. 1). El lenguaje no solamente es plenamente colectivo sino que, según afirma Gutiérrez (2006), "en el diálogo, se desarrolla una 'zona de sensibilidad' que trasciende lo que normalmente reconocemos como pensar. Esta sensibilidad es una 'red sutil' capaz de recoger los significados sutiles del flujo del pensar" (p. 9).

\section{¿Donde perdimos el sentido de la vida?}

La vida se colorea de sentido cuando el ser humano tiene noción de que viene a este mundo a pasar por un proceso de aprendizaje; que la vida también tiene algún sentido y que todo está en constante interrelación. El primer estudioso que habló de estados superiores de consciencia fue Karl Jung, psiquiatra y discípulo de Freud, quien en clara oposición al modelo sexualizado y centrado en patologías del psicoanálisis, empieza a considerar otra realidad que, posteriormente, ofreció los fundamentos de la psicología transpersonal. Esta escuela nace a finales de la década de los sesenta y, como su nombre lo indica, va más allá de la personalidad, 
abandona la enfermedad y centra su atención en el ser humano saludable, creativo, gozoso, espléndido y feliz. A este cambio paradigmático centrado en una psicología de personas sanas, se une una mayor apertura hacia las enseñanzas de Oriente, con lo que se profundiza también en temas como la meditación, el yoga y el vegetarianismo.

Esta nueva visión de vida llega a la dimensión espiritual del ser humano, entendiendo la espiritualidad como el reconocimiento desde la experiencia de que somos parte de algo transcendente y superior. Lo cual, hasta ese momento era considerado por el viejo paradigma psicoanalítico como una sublimación de lo inferior, posición en la cual si yo estoy aquí y el otro está allá, el que está allá está perturbado. Por el contrario, la psicología transpersonal centra el interés esencialmente en las personas que trabajan en la producción de estados no ordinarios de consciencia, estados de mayor lucidez mental, de amor incondicional e investiga estados internos como el dormir, el soñar y la compasión profunda.

La psicología transpersonal, además de tomar los aportes de la psicología actual, recupera el conocimiento antiguo de las tradiciones y culturas ancestrales, apoyándose en distintas tradiciones como la psicología budista, la taoísta, la psicología de nativos de diferentes partes del mundo y las asume más allá del aspecto religioso, para fundamentar una filosofía que expone que el ser humano tiene en sí mismo una fracción de lo divino, la cual en muchos casos nunca es descubierta.

Contrapuesto a todo lo mencionado hasta acá, cuando revisamos lo que estamos haciendo en los procesos educativos formales, podríamos decir que, en muchos casos, es eliminar toda búsqueda del sentido y de la trascendencia. Probablemente en el tipo de educación actual estemos lejos de ayudarle a la persona a valorar lo bello que tiene en sí misma y a desplegarlo en su vida cotidiana, en su familia, en su pareja y en las tareas creativas. La educación existente difícilmente llega a motivar a la persona para que se anime y se permita ser, porque -como se mencionó al inicio- ni el mismo personal docente se presenta, en muchos casos, como es. Los procesos educativos así vividos nunca llevarán a la búsqueda de lo divino que encierra nuestro ser.

Olvidamos, en estos procesos, lo que antes mencionábamos y que algunos autores han llamado poéticamente el "manto sin costuras del universo"; es decir, hay unidad, todos somos uno $y$, actualmente, incluso es posible identificar puntos de encuentro entre la filosofía perenne y la física cuántica.

Existe la hipótesis de que hay un tipo de información compartida en cada especie y que se puede participar de ese aprendizaje gracias a lo que Sheldrake (1994) define como resonancia mórfica, la cual, aunque parezca un concepto extraño, involucra la simpleza de la verdad. Él habla de campos de conciencia en donde todos los seres estamos interrelacionados. Estos campos están constituidos por las formas y las actitudes de todos los individuos pasados de una especie y su influencia moldearía a los individuos futuros, en algo como un tipo de memoria colectiva. 
doi: http://dx.doi.org/10.15359/ree.18-3.16

URL: http://www.una.ac.cr/educare

CORREO: educare@una.cr

La búsqueda del sentido nos lleva a reflexionar acerca de: ¿qué se puede encontrar que está escondido? Muchas tradiciones antiguas nos hablan del viaje del héroe; para ellas el héroe es cada uno y cada una de nosotras buscando nuestro propio sentido y, parte de esa búsqueda, tiene que ver con un tesoro escondido que probablemente vamos encontrando poco a poco. Lo imaginamos muy personal, pero este tesoro, a la vez, es compartido porque la persona que busca encuentra en el camino a otros $y$, en este proceso, nos vamos re-conociendo.

La psicología transpersonal está centrada en el ser, en ese núcleo esencial que vino a este mundo a desplegarse y que conoce muy bien a lo que vino. De Jung podemos rescatar la noción de que mi ser tiene la idea de desplegar a ser lo máximo que vino a ser. El meollo es escucharme para saber qué vine a ser, eliminando todas las voces que nos exigen ser lo que no somos y, dentro de mí, siempre estará eso que me responda: donde el silencio es el mejor medio para escuchar-lo.

Muchas personas cumplen, a lo largo de su vida, con todas las demandas sociales que les fueron impuestas desde que nacieron: ingresaron a la escuela, a la universidad, a la vida laboral, a la vida en pareja; pero luego se percatan de que no hicieron muchas de las cosas que quisieron realmente hacer; es decir, no escucharon su núcleo esencial. Es frecuente que esto se observe a mitad de la vida de una persona, cuando ya el cuerpo no es el mismo y muchos seres queridos ya no están, entonces puede surgir la temida crisis de los años maduros. No obstante, esta crisis debería verse como una valiosa oportunidad para quebrar con esa cáscara de la que hablamos desde el inicio y que es construida por toda la fragmentación y todas las programaciones que soportamos. Es el momento, entonces, para re-crearse partiendo de la crisis.

\section{La penúltima pregunta sin respuesta, a manera de conclusión}

¿Qué hacemos para recuperar todo lo que nuestros antepasados comprendían, sentían y vivían claramente y en la actualidad hay que trabajar duro para medianamente alcanzarlo?

Aunque no pretendo encontrar la respuesta, la educación debería replantear su concepto de vida y del sentido de la vida, centrándose en ese núcleo esencial del que hemos hablado; en lo sagrado que hay en mí y en cada una de las personas con quienes comparto los espacios y los momentos áulicos. Debo aprender a "saborearlo" como una esencia que viene a desplegarse, a desarrollarse para sacar y compartir lo que está oculto. No trabajar con el intelecto porque en realidad este es solo una herramienta; más bien se trata de una experiencia y no de explicar qué es un estado superior o un estado de armonía. Un nuevo paradigma en el que se trabaje la totalidad de la vida: corazón y razón y, por tanto, la vida misma.

Concluyo con una cita de Gutiérrez (2006), la cual reviste más sentido cada vez que la leo: "Nada debe hacer el estudiante o la estudiante si para eso que tienen que hacer no le encuentran sentido" (p. 4). 


\section{Referencias}

Assmann, H. (2002). Placer y ternura en la educación. Hacia una sociedad aprendiente. Madrid: Narcea Ediciones.

Castaneda, C. (2001). El lado activo del infinito. México: Punto de lectura.

Gutiérrez, F. (2006). Doctorado de la tercera cultura: En busca del sentido. San José, Costa Rica: Universidad de La Salle.

Maturana, H. (2002). Transformación en la convivencia. Santiago: Dolmen Ediciones.

Maturana, H. y Varela, F. (1998). De máquinas a seres vivos. Autopoiesis: La organización de lo vivo. Santiago: Editorial Universitaria.

Ruiz, A. (1997). Las contribuciones de Humberto Maturana a las ciencias de la complejidad y a la psicología. Santiago: Instituto de Terapia Cognitiva INTECO. Recuperado http://www. inteco.cl/articulos/005/texto esp.htm

Sheldrake, R. (1994). El renacimiento de la naturaleza: La nueva imagen de la ciencia y de Dios. Barcelona: Paidós.

\section{Cómo citar este artículo en APA:}

Miranda-Cervantes, G. (setiembre-diciembre, 2014). Reflexiones en torno al sentido de la vida en los espacios áulicos. Revista Electrónica Educare, 18(3), 263-271. doi: http://dx.doi.org/10.15359/ree.18-3.16

Nota: Para citar este artículo en otros sistemas puede consultar el hipervínculo "Como citar el artículo" en la barra derecha de nuestro sitio web: http://www.revistas.una.ac.cr/index.php/EDUCARE/index 